\title{
Directed transport with real-time steering and drifts along pre-designed paths using a Brownian motor
}

\author{
H. Hagman, ${ }^{1},{ }^{*}$ M. Zelan, ${ }^{1}$ C. M. Dion, ${ }^{1}$ and A. Kastberg ${ }^{2}$ \\ ${ }^{1}$ Department of Physics, Umeå University, SE-901 87 Umeå, Sweden. \\ ${ }^{2}$ Laboratoire de Physique de la Matière Condensée, CNRS UMR 6622, \\ Université de Nice-Sophia Antipolis, Parc Valrose, F-06108 Nice Cedex 2, France
}

(Dated: October 31, 2018)

\begin{abstract}
We have realized real-time steering of the directed transport in a Brownian motor based on cold atoms in optical lattices, and demonstrate drifts along pre-designed paths. The transport is induced by spatiotemporal asymmetries in the system, where we can control the spatial part, and we show that the response to changes in asymmetry is very fast. In addition to the directional steering, a real-time control of the magnitude of the average drift velocity and an on/off switching of the motor are also demonstrated. We use a non-invasive real-time detection of the transport, enabling a feedback control of the system.
\end{abstract}

PACS numbers: 05.40.-a, 05.60.-k, 37.10.Jk

\section{INTRODUCTION}

Many microscopic systems have a noisy dynamic governed by thermal fluctuations, making their control and theoretical treatment complicated. However, Brownian motors and Brownian ratchets take advantage of this noise as they convert random fluctuations into directed motion in the absence of bias forces [1-4]. This makes them an interesting subject of statistical physics, and they are the driving mechanisms of a variety of biological motors [1, 5], ranging from inter-cell transport and virus translocation to muscle contraction [6]9]. Inspired by these biological machines, several proposals exist to utilize the principles of Brownian motors to power up future nano-technology [10, 11. Many actual Brownian motors are relatively large and complex systems, therefore models with more comprehensive and controllable designs are needed [1, 4, 5. Beside the naturally occurring biological motors a number of artificial Brownian motors and ratchets have been realized, such as cold atom Brownian motors and ratchets [12 15] and quantum ratchets [1618.

For a directed motion to be induced in any of these systems two requirements have to be fulfilled: the spatial and/or temporal symmetries have to be broken, in accordance with the Curie principle [19, and the system has to be brought out of thermal equilibrium, in agreement with the second law of thermodynamics 20. Fulfilling these requirements is sufficient to induce drifts, which is well established, and a number of different types of systems have been demonstrated 4, 21. Reversals of the induced drift have also been demonstrated, e.g., [22], but the response to an asymmetry changing in real time, and in three dimensions, hasn't been fully investigated. All these aspects can be addressed by Brownian motor systems realized with laser cooled atoms interacting with three-dimensional (3D) dissipative optical lattices 23]. These make a promising testbed for fundamental studies of the underlying mechanisms in play [4, and they can be utilized as proof of principle experiments, or as experimental demonstrations of the feasibility of Brownian motors.

We here demonstrate a Brownian motor, realized with cold atoms interacting with two optical lattices, where the asymmetry, and hence the average drift of the atoms, is controllable in real time in 3D. The optical lattices are individually symmetric, and the required asymmetry originates instead from a combination of a relative translation of, and unequal transfer rates between, the optical lattices. This gives a flexible setup where the rectification can be controlled via the relative translation of the lattice potentials [13. We here introduce an external real-time control of the translation of the lattices, by use of electro-optical modulators, along with real-time, nondestructive measurement by fluorescence imaging. This allows us to investigate the response of the system to a changing asymmetry, as well as the possibility to utilize these asymmetry changes for external real-time steering of the induced drift, and for inducing of drifts along predesigned paths.

\section{WORKING PRINCIPLE}

To qualitatively understand the induced drift dependence on the translation between the potentials, a simple 1D model is used 24, see Fig. 1. Consider a classical Brownian particle situated in either of two symmetric and periodic potentials (S and L). Both potentials have an inherent friction and Brownian diffusion, and are coupled with unequal transfer rates. The particle randomly switches potential, spending a longer time in potential L. When the potentials are in phase, the particle will mostly be located near the bottom of a well, undisturbed by the inter-potential transfer, see Fig. 1 $\mathrm{b}$. The system is symmetric and no drift is induced. However, if one 
potential is translated with respect to the other (a relative phase shift $\varphi$ ), the spatio-temporal symmetry is broken, and the passage from one potential to the other, on average, adds energy to the particle [25]. This gain in energy, together with the relative translation, leads to an increased diffusion, which due to the total asymmetry is biased (Fig. 1, ), with maximum drift occurring around $\varphi= \pm 2 \pi / 3$. A translation of half a period only increases the unbiased diffusion since the symmetry is restored, see Fig. 11d. Although it contains all the essential ingredients of the Brownian motor, this model is an simplification of the experimental system [13, 26]. For cesium atoms, each of the two potentials is a manifold of potentials of different amplitudes 23., of which one dominates the dynamics. Moreover, the damping force applied to an atom, and the transfer rates between the potentials, are position and velocity dependent [23, 27.
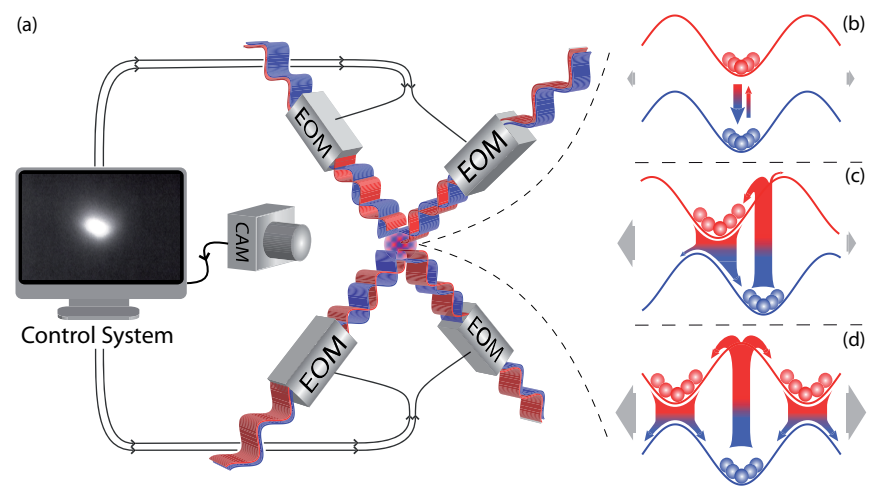

FIG. 1: (Color online) (a) Schematic representation of the key elements of the experimental set-up. The two optical lattices are constructed from the interference patterns from two superposed, four-laser-beam configurations. In each arm a computer controlled EOM is located, changing the relative phase $\varphi$. The fluorescence of the atoms is monitored by the control system through a CCD camera. (b,c,d): 1D representation of the atoms in the long-lived (lower, blue) and the short-lived (upper, red) optical lattices. In each potential an inherent friction and diffusion is present. The vertical arrows indicate the transfer between the potentials, and the horizontal the total diffusion. (b) $\varphi=0$, the system is symmetric and the particles are mostly located near the bottom of the wells, undisturbed by the inter-potential transfer. No drift is induced. (c) $\varphi=2 / 3 \pi$, the transfer adds energy to the system, the symmetry is broken, and a drift to the left is induced. (d) $\varphi=\pi$, the overall symmetry is restored, and the added energy only gives an increased, unbiased diffusion.

\section{EXPERIMENTAL SETUP}

Detailed descriptions of the experimental setup are given in [13, 27]. In short, about $10^{8}$ cesium atoms are accumulated and cooled to around $5 \mu \mathrm{K}$ in a magnetooptical trap 28]. The latter is then switched off, and two optical lattices 23] are superposed on the atoms. These optical lattices are 3D light-shift potentials, created in the interference pattern of laser beams, and are dissipative [23], that is, they are constructed from light fields which are tuned sufficiently close to an atomic resonance for light scattering to be important, providing a source of noise as well as an inherent friction due to laser cooling [23, 27. Moreover, the two optical lattices are spatially overlapped, have the same periodicity, and an atom interacts with either depending on its hyperfine state. The incoherent light scattering provides a route between the two optical lattices, via a manifold of short-lived excited states. The transfer rates are highly unequal, which results in one short-lived and one long-lived optical lattice (denoted S and L, respectively). The potential depths correspond to around $100 \mu \mathrm{K}$ in lattice $\mathrm{S}$ and $200 \mu \mathrm{K}$ in lattice $\mathrm{L}$, while the kinetic temperature of the atoms is around $10 \mu \mathrm{K}$. The kinetic temperature has a dependence on the relative translation of the potentials [27, and can therefore be used as a measurement of this translation, or for monitoring the relaxation to steady state after a change in the system.

In contrast to previous work, the relative translation of the optical lattices is now controlled with electro-optical phase modulators (EOMs). That is, the optical lattice configuration consists of four arms, each with two laser beams with orthogonal polarizations. An EOM is placed in each arm to control the relative phase of the two beams. The crystals inside the EOMs have one electrooptical active axis and one passive axis. Along the active axis, the index of refraction is dependent on an externally applied voltage allowing the external control of the optical path length, while along the passive axis it remains unchanged. By controlling the relative optical phase of each arm, the relative spatial phase of the resulting potentials can be controlled as well. After the EOMs, but before the arms start to interfere, the polarization of the two beams in each arm are turned to the same direction. The setup is also modified to image the atoms through the inherent fluorescence in the optical lattices (due to light scattering), such that the detection doesn't interfere with the system. The current experimental setup is illustrated schematically in Fig. 19.

\section{RESULTS}

To experimentally investigate the real-time response of the Brownian motor to changes in the relative translation of the optical potentials, $\left(\varphi_{x}, \varphi_{y}, \varphi_{z}\right)$, we start with translations in 1D. This is done in five steps: $(0,0,0) \rightarrow$ $(2 \pi / 3,0,0) \rightarrow(-2 \pi / 3,0,0) \rightarrow(2 \pi / 3,0,0) \rightarrow(0,0,0)$, see Fig. $2 \mathrm{a}$ where a selection of images, representing the extreme points of the trajectory, are presented. The whole trajectory is available online as movie S1 29. The cloud is imaged in the xz-plane, with $z$ being vertical in the experiment, but horizontal in the images. In the figure, the 
expected back and forth trajectory in $x$ is evident. Beside the back and forth motion, a small downwards drift is also present, since the optical lattices can't fully support the atomic cloud against gravity 30. In principle this effect could be canceled by an imbalanced radiation pressure, or by an appropriate choice of $\varphi_{z}$. In Fig. 2 $2 \mathrm{~b}$, the time evolution of the atomic cloud's center of mass along $x$ is presented. The drift velocities are constant for a fixed translation, and the change in direction when the potentials are translated appears to be very fast. To further investigate this, we have repeated the same set of relative potential translations, but on a shorter time scale, and measured the temperature using a (destructive) time-of-flight technique [31. Figure 2k shows that the reaction time for the atoms to reach the new steady state is less than $1 \mathrm{~ms}$, i.e., is not resolvable, given the time resolution and uncertainty of our control and detection systems.
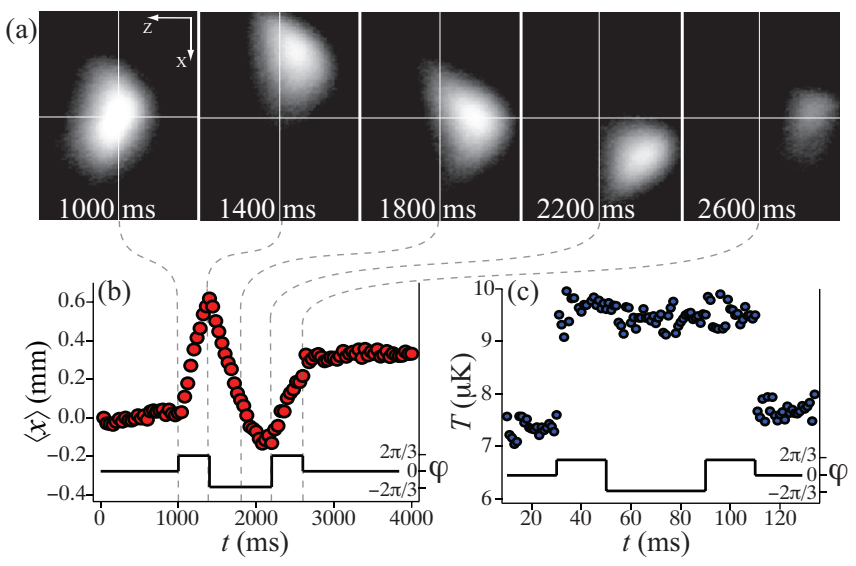

FIG. 2: (Color online) External steering of the induced drift in 1D. The translation between the potentials, $\left(\varphi_{x}, \varphi_{y}, \varphi_{z}\right)$, are changed according to the sequence $(0,0,0) \rightarrow(2 \pi / 3,0,0) \rightarrow$ $(-2 \pi / 3,0,0) \rightarrow(2 \pi / 3,0,0) \rightarrow(0,0,0)$. (a) Selected images of the atomic cloud in the xz-plane, with $z$ directed upwards in the experiment. (b) Time evolution of the center-of-mass position along $x$. (c) Time evolution of the temperature for the same sequence of translational shifts, but on a shorter time scale. In the lower part of (b) and (c) the phase sequences are plotted.

The relative translation of the potentials can also be altered in other directions, making it possible to move the atoms along arbitrary pre-designed paths, including closed figures. We first make the atoms move along paths with straight angles, as the relative phases of the potentials are changed in four steps: $(2 \pi / 3,0,0) \rightarrow$ $(0,-2 \pi / 3,0) \rightarrow(-2 \pi / 3,0,0) \rightarrow(0,2 \pi / 3,0)$. The interval between each shift is around $300 \mathrm{~ms}$. Figure $3 \mathrm{a}$ (and movie S2 29]) show the expected square path.

Since drifts in arbitrary directions are achievable, any geometrical figure can in principle be realized. Figure 3b (and movie S3 29]) demonstrates the guiding of the atoms along a triangular path, achieved by rel-
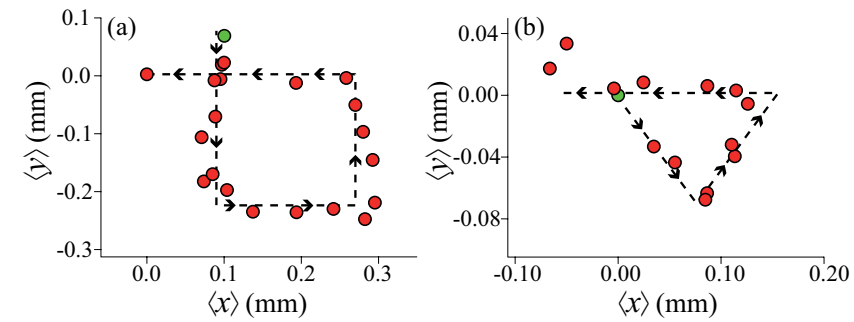

FIG. 3: (Color online) Drifts along pre-designed closed paths: (a) square; (b) triangle. The trajectory of the center of mass is marked with filled circles, starting from the green (light gray) one, with the anticipated path indicated by a dashed line. The time interval between each marker is roughly 75 ms.

ative phase settings of $(2 \pi / 3,0,0) \rightarrow(-\pi / 3,-\pi / 3,0) \rightarrow$ $(-\pi / 3, \pi / 3,0)$. Note that the phase settings for off-axis drifts become non-trivial due a coupling between the dimensions of the lattice topography [26].

All data where taken for relative translations of the potentials that optimize the magnitude of the drift. It can however be made arbitrary small [13, 26], giving us not only real-time control of the direction, but also of the speed of the atoms. In addition, the directional shifts do not need to be discrete, as the electro-optical modulators can be controlled continuously. Smooth velocity changes are thus achievable, allowing for curved paths.

With the exception of Fig. 2k, all the results presented are recorded by imaging the inherent fluorescence of the atomic cloud in the optical lattices. Therefore the detection does not interfere with the system, enabling a realtime analysis of the position of the atomic cloud. This opens up the possibility of implementing a feedback loop, that is, an autonomous system can be created, where the atomic cloud's current position and velocity determines the system's coming actions.

\section{CONCLUSION}

In summary, we have realized a Brownian motor with a real-time external steering in $3 \mathrm{D}$, and demonstrated drifts along pre-designed paths. The directional shifts of the average velocity was shown to be fast $(<1 \mathrm{~ms})$, with induced average velocities are up to a few $\mathrm{mm} / \mathrm{s}$, while the lifetime of the atoms in the optical lattice is of the order of seconds. This gives ample time to induce drifts with several resolvable directional shifts, see movie S4 29]. On a more general scale, a typical velocity of $1 \mathrm{~mm} / \mathrm{s}$ means that each atom, on average, moves over 1600 potential wells per second. We showed that the induced drifts can be detected in real time and nondestructively, opening possibilities for feedback control of the induced drift. The system could hence be controlled externally in real time by, e.g., a joystick, by 
pre-determine protocols inducing drifts along designed paths, or via a position feedback that enables the system to autonomously steer itself. The feedback could be used, e.g., to stabilize drifts or for the system to find its own way through a maze. Real-time steering, drifts along pre-designed paths, and feedback controlled drifts are all important for the creation of useful future applications of Brownian motors [10, 11, 32. Our system may also function as a model system that can be used for fundamental studies of the properties and feasibility of Brownian motors.

We thank S. Jonsell and G. Labaigt for helpful discussions. This project was supported by the Swedish Research Council, Knut \& Alice Wallenbergs Stiftelse, Carl Trygger Stiftelse, Kempe Stiftelserna, and Umeå University.

* Electronic address: henning.hagman@physics.umu.se

[1] R. D. Astumian, Science 276, 917 (1997).

[2] P. Reimann, Phys. Rep. 361, 57 (2002).

[3] P. Hänggi, F. Marchesoni, and F. Nori, Ann. Phys. (Leipzig) 14, 51 (2005).

[4] P. Hänggi and F. Marchesoni, Rev. Mod. Phys. 81, 387 (2009).

[5] F. Jülicher, A. Ajdari, and J. Prost, Rev. Mod. Phys. 69, 1269 (1997).

[6] B. Alberts, Cell 92, 291 (1998).

[7] R. D. Vale and R. A. Milligan, Science 288, 88 (2000).

[8] R. D. Vale, Cell 112, 467 (2003).

[9] G. Oster and H. Wang, Trends in Cell Biology 13, 114 (2003).

[10] M. G. L. van den Heuvel and C. Dekker, Science 317, 333 (2007).

[11] J. Wang, ACS Nano 3, 4 (2009).

[12] C. Mennerat-Robilliard, D. Lucas, S. Guibal, J. Tabosa, C. Jurczak, J.-Y. Courtois, and G. Grynberg, Phys. Rev. Lett. 82, 851 (1999).

[13] P. Sjölund, S. J. H. Petra, C. M. Dion, S. Jonsell,
M. Nylén, L. Sanchez-Palencia, and A. Kastberg, Phys. Rev. Lett. 96, 190602 (2006).

[14] P. H. Jones, M. Goonasekera, D. R. Meacher, T. Jonckheere, and T. S. Monteiro, Phys. Rev. Lett. 98, 073002 (2007).

[15] A. B. Kolton and F. Renzoni, Phys. Rev. A 81, 013416 (2010).

[16] H. Linke, T. E. Humphrey, A. Löfgren, A. O. Sushkov, R. Newbury, R. P. Taylor, and P. Omling, Science 286, 2314 (1999).

[17] J. B. Majer, J. Peguiron, M. Grifoni, M. Tusveld, and J. E. Mooij, Phys. Rev. Lett. 90, 056802 (2003).

[18] T. Salger, S. Kling, T. Hecking, C. Geckeler, L. MoralesMolina, and M. Weitz, Science 326, 1241 (2009).

[19] P. Curie, J. Phys. III (Paris) 3, 393 (1894).

[20] R. P. Feynman, R. B. Lieghton, and M. Sands, The Feynman Lectures on Physics, vol. 1 (Addison-Wesley, Reading, MA, 1963).

[21] Special issue on Ratchets and Brownian Motors: Basics, Experiments and Applications, H. Linke, Ed., Appl. Phys. A 75 (no. 2) (2002).

[22] C. C. de Souza Silva, J. V. de Vondel, M. Morelle, and V. V. Moshchalkov, Nature 440, 651 (2006).

[23] G. Grynberg and C. Robilliard, Phys. Rep. 355, 335 (2001).

[24] L. Sanchez-Palencia, Phys. Rev. E 70, 011102 (2004).

[25] D. Suzuki and T. Munakata, Phys. Rev. E 68, 021906 (2003).

[26] H. Hagman, C. M. Dion, P. Sjölund, S. J. H. Petra, and A. Kastberg, EPL 81, 33001 (2008).

[27] H. Ellmann, J. Jersblad, and A. Kastberg, Phys. Rev. Lett. 90, 053001 (2003).

[28] H. J. Metcalf and P. van der Straten, J. Opt. Soc. Am. B 20, 887 (2003).

[29] See supplemental material for movies of the drift of the atomic cloud in various cases.

[30] M. Zelan, H. Hagman, K. Karlsson, C. M. Dion, and A. Kastberg, Phys. Rev. E 82, 031136 (2010).

[31] H. Hagman, P. Sjölund, S. J. H. Petra, M. Nylén, A. Kastberg, H. Ellmann, and J. Jersblad, J. Appl. Phys. 105, 083109 (2009).

[32] H. Hess, G. D. Bachand, and V. Vogel, Chem. Eur. J. 10, 2100 (2004). 